\title{
Restoration of Oral Function for Adult Edentulous Patients with Ectodermal Dysplasia: A Prospective Preliminary Clinical Study
}

\author{
Yiqun Wu, PhD, MD;* Xu Dong Wang, PhD, MD; Feng Wang, MD;* Wei Huang, MD;* \\ Zhiyong Zhang, MD; ${ }^{*}$ Zhiyuan Zhang, $\mathrm{PhD}, \mathrm{MD} ;^{\dagger}$ Darnell Kaigler, $\mathrm{PhD}, \mathrm{DDS} ;^{\ddagger}$ Duohong Zou, $\mathrm{PhD}, \mathrm{MD}^{\S}$
}

\begin{abstract}
Background: Therapy with zygomatic implants (ZIs) or conventional implants (CIs) has proven to be an effective method to restore oral function for systemically healthy patients. However, it is still a major challenge to fully restore oral function to edentulous adult patients with ectodermal dysplasia (ED).
\end{abstract}

Purpose: The aim of this study was to determine an effective treatment protocol for restoring oral function using ZIs and CIs to edentulous adult ED patients.

Materials and Methods: Ten edentulous adult ED patients were treated in this study. The treatment protocol involved the following: (1) bone augmentation in the region of the anterior teeth; (2) placement of two ZIs and four CIs in the maxilla, and four CIs in the mandible; (3) fabrication of dental prosthesis; and (4) psychological and oral education. Following treatment of these patients, implant success rates, biological complications, patient satisfaction, and psychological changes were recorded.

Results: Although there was evidence of bone graft resorption in the maxilla, bone augmentation of the mandible was successful in all patients. Nine CIs in the maxilla failed and were removed. All ZIs were successful, and the CIs success rates were $77.50 \%$ in the maxilla and $100 \%$ in the mandible, with a mean of $88.75 \%$. The mean peri-implant bone resorption for the CIs ranged from $1.3 \pm 0.4 \mathrm{~mm}$ to $1.8 \pm 0.6 \mathrm{~mm}$, and four cases exhibited gingival hyperplasia in the maxilla and mandible. One hundred percent of the patients were satisfied with the restoration of their oral function, and $>50 \%$ of the patients exhibited enhanced self-confidence and self-esteem.

Conclusions: This study demonstrates that oral function can be restored in edentulous adult ED patients using a comprehensive and systematic treatment protocol involving psychological and oral education, bone augmentation, implant placement, and denture fabrication. Despite these positive outcomes, bone augmentation remains challenging in the anterior region of the maxilla for edentulous adult ED patients.

KEY WORDS: bone augmentation, ectodermal dysplasia, oral function reconstruction, severe bone defects, vertical distraction osteogenesis, zygomatic implants

\footnotetext{
${ }^{\star}$ Professor, Department of Oral Implant, School of Medicine, Ninth People's Hospital Affiliated with Shanghai Jiao Tong University, Shanghai, China; ${ }^{\dagger}$ Professor, Department of Oral and Maxillofacial Surgery, School of Medicine, Ninth People's Hospital Affiliated with Shanghai Jiao Tong University, Shanghai, China; ${ }^{*}$ Assistant professor, Department of Periodontics and Oral Medicine, Department of Biomedical Engineering, Michigan Center for Oral Health Research, University of Michigan, Ann Arbor, MI, USA; ${ }^{\S}$ Associate professor, Department of Dental Implant Centre, Stomatologic Hospital \& College, Key Laboratory of Oral Diseases Research of Anhui Province, Anhui Medical University, Hefei, China
}

Corresponding Author: Prof. Yiqun Wu, Department of Oral Implant, School of Medicine, Ninth People's Hospital Affiliated with Shanghai Jiao Tong University, 639 Zhi Zao Ju Road, Shanghai 200011, China; e-mail: yiqunwu@hotmail.com; Prof. Duohong Zou, Department of Dental Implant Centre, Stomatologic Hospital \& College, Key Laboratory of Oral Diseases Research of Anhui Province, Anhui Medical University, 69 Meishan Road, Hefei 230032, China; e-mail: zouduohongyy@126.com

(C) 2015 Wiley Periodicals, Inc.

DOI 10.1111/cid.12296 


\section{INTRODUCTION}

Ectodermal dysplasia (ED) is a group of heritable disorders that cause the hair, teeth, nails, and glands to develop and function abnormally. ${ }^{1} \mathrm{Up}$ to seven of every 10,000 babies are affected by ED. ${ }^{2}$ According to the degree of sweat gland function, ED is mainly divided into two types: hypohidrotic ED (HED) or hidrotic ED. ${ }^{3,4}$ HED is the most common ED condition. ${ }^{1,5}$ ED symptoms range from mild to severe. The severe clinical symptoms include multiple tooth abnormalities (anodontia and hypodontia), severe alveolar ridge atrophy, hypotrichosis (fine, sparse blond hair, including decreased density in both eyebrows and eyelashes), a prominent forehead and chin, deficient tears and saliva, poorly functioning mucous membranes, thick and protruding lips, "saddle" nose, hearing or vision deficits, sensitivity to light, missing fingers or toes, cleft lip or palate, immune system disorders, and other ectoderm abnormalities. ${ }^{6,7}$ These manifestations impact many facets of the daily lives of ED patients, including work, social activities, and overall physiological and psychological well-being. Adult ED patients with anodontia often exhibit severe alveolar ridge atrophy and problems with mastication, speech, and facial appearance. Thus, restoration of oral form and function in these patients can be life changing and is the goal of dental therapy.

Dental implants have been used to support complete dentures for decades; therefore, they have become a predictable modality to restore oral function to systemically healthy edentulous patients. In 1998, Brånemark introduced a zygomatic implant (ZI), which is placed in the lateral orbital rim and zygomatic arch. ${ }^{8}$ ZIs provide a good alternative to rehabilitate the atrophic maxilla in patients with insufficient bone volume. However, it is still challenging for dentists to restore oral function in edentulous adult ED patients due to severe alveolar bone atrophy. ${ }^{9}$ Clinically, several methods have been used to augment bone volume, including the use of vertical distraction osteogenesis (DO), autogenous bone grafts, allogeneic bone grafts, xenogeneic bone grafts, and combinations of these modalities.

Since it was first adopted to augment bone volume in the craniomaxillofacial region in 1992, DO has played an important role in managing congenital bone defects as well as defects resulting from surgical resection or trauma. ${ }^{10}$ When DO was extended to the augmentation of alveolar bone, the technique was called alveolar DO
(ADO). In 1996, ADO therapy was documented to be an effective method of augmenting bone volume in the jaw. ${ }^{11}$ Compared with other bone volume augmentation techniques, $\mathrm{ADO}$ has several key advantages, including (1) a decreased likelihood of graft resorption; (2) a more predictable gain of hard and soft tissue volume; (3) a shorter bone consolidation/integration period reducing the total treatment time; (4) the potential for teeth or implants to be included in the transported fragment to ensure that occlusal or esthetic defects can be corrected; (5) the use of complimentary regeneration techniques when the outcome is not completely satisfactory; and (6) no morbidity associated with a secondary surgical donor site. ${ }^{12}$ Though advantages exist, ADO also has limitations associated with certain complications, such as infection in the distraction chamber, fractures of transported or basal bone, premature consolidation, wound dehiscence, consolidation delays, and the potential formation of a fibrous nonunion. ${ }^{12}$ More importantly, the distractor stability is important for ADO success.

In edentulous $\mathrm{ED}$ patients, the alveolar bone in the anterior region of the maxilla can be "knife-edge" thin. To address this problem, autogenous, allogeneic, and xenogeneic bone grafts are also often used to enhance bone volume in the anterior region of the maxilla. Clinically, autogenous bone grafts of the fibula, ilium, or scapula have been successfully developed to address the requirements for bone height and width of the alveolar ridge. ${ }^{13}$ Of these graft types, relative to scapula bone grafts, the fibula and the iliac crest were both shown to be the best options for large facial reconstruction and provide adequate bone volume for implant placement. ${ }^{14}$ In this prospective study, we chose a comprehensive method to augment alveolar bone of the maxilla and mandible in edentulous ED patients. Autogenous fibula or ilium grafts were used to augment bone volume in the area of the anterior teeth in the maxilla, and ADO was used to augment the bone volume in the area of the anterior teeth in the mandible.

Previous reports have demonstrated that conventional implants (CIs) and ZIs can be used to restore oral function to healthy patients or ED patients following bone augmentation. ${ }^{8,15-19}$ However, being that these studies are case reports, there is no standardized treatment for edentulous ED patients. In addition, there have been few reports which incorporate patient-centered modalities into therapy, such as psychological variables 
and oral education. Therefore, the objective of this prospective study tried to establish a comprehensive, standardized, and predictable protocol for restoring oral function in adult ED patients with severe bone atrophy. This treatment protocol included psychological education, bone augmentation, choice of implants, and patient education on oral health.

\section{MATERIALS AND METHODS}

\section{Patient Selection}

From January 2007 to April 2011, 10 ED patients with edentulous jaws were selected to participate in this prospective clinical study at the Department of Oral and Maxillofacial Surgery and the Department of Oral Implants, Ninth People's Hospital Affiliated with Shanghai Jiao Tong University, School of Medicine, Shanghai, China. The following inclusion criteria were applied: (1) ED patients with edentulous jaws and age $\geq 18$ years; (2) patients with severe alveolar ridge atrophy (alveolar bone height $<5 \mathrm{~mm}$ ); (3) patients who chose to restore oral function with implants; (4) augmented bone volume (ADO used in the mandible and autogenous bone grafting used in maxilla.); (5) ZIs placed in the site of the lateral orbital rim, and zygomatic arch and CIs placed in the site of the anterior teeth in the jaw; (6) two ZIs placed in the zygomatic bone and four CIs placed in the anterior area of maxilla as well as four CIs placed in the anterior area of the mandible; and (7) patients who agreed to regular follow-up and psychological and oral education for the duration of the study. The exclusion criteria included any uncontrolled systemic or neurological disease and current smokers. Ten patients (three female and seven male, between 18 and 25 years old, average age 20.1 years) fulfilled these criteria and were enrolled in the study (Table 1). All patients provided informed consent to participate in this study.

\section{Bone Augmentation}

All patients underwent bone augmentation procedures. Autologous iliac crest or fibula grafts were used in the anterior region of the maxilla. Under general anesthesia, an alveolar ridge incision was created slightly palatal and extended from the right primary first molar to the contralateral primary first molar in the upper jaw with one vertical releasing incision in the median line posteriorly. In general, reflection of the labial/buccal flap was full thickness. After adequately exposing the alveolar bone, autogenous bone blocks from the iliac crest or fibula

\begin{tabular}{lc}
\hline TABLE 1 Patients' Characteristics of the Study \\
\hline Characteristics & Patients $(n=10)$ \\
\hline Mean age in years (SD) & $20.1(1.2)$ \\
Gender (M/F) & $7 / 3$ \\
Implants $(n)$ & ZIs $=20 ;$ CIs $=80$ \\
Length & ZIs $=40,52.5 \mathrm{~mm} ;$ \\
& ITI $=10,12 \mathrm{~mm} ;$ \\
& Nobel biocare $=11$, \\
& $13 \mathrm{~mm}$ \\
Diameter & $4.0 \mathrm{~mm} ; 4.1 \mathrm{~mm} ;$ \\
& $4.3 \mathrm{~mm}$ \\
Bone augmentation in & ADO $=10$ \\
mandible & Iliac graft $=6 ;$ \\
Bone augmentation in & fibular graft $=4$ \\
maxilla & $1.2(0.4)$ \\
Mean reconstruction & Implant-supported \\
period in years $(\mathrm{SD})$ & fixed dentures $=19 ;$ \\
Type of restoration & implant-supported \\
& overdenture $=1$ \\
&
\end{tabular}

$\mathrm{ADO}=$ alveolar distraction osteogenesis; $\mathrm{CI}=$ conventional implant; $\mathrm{F}=$ female; $\mathrm{M}=$ male; $\mathrm{ZI}=$ zygomatic implant.

were grafted to the lateral wall of the maxilla using an onlay technique. The guided bone regeneration (GBR) technique was next used to facilitate bone augmentation in the area of implant placement surrounding the fixed blocks using Bio-Oss xenogeneic bone and a Bio-Gide membrane (Bio-Gide, Geistlich, Wolhusen, Switzerland). Finally, a tension-free primary wound closure was achieved with interrupted and mattress sutures. For the mandible, ADO was performed in the interforaminal region of the mandible. The ADO surgery was completed as previously described. ${ }^{20}$ Briefly, an incision was created in the vestibular position for protecting the lingual mucoperiosteum of the distracted bone segment. The mental foramen was identified and exposed to avoid damaging the nerve during the ADO procedure. According to the configuration of the labial bone at the mandibular symphysis, an appropriate distraction device was selected. Vertical osteotomies were securely completed in the interforaminal region of the mandible. The distal ends of the fixation plates were then securely fixed with microscrews. Finally, the mobility of the distraction bone segment was confirmed using the distraction screw, and the soft tissue wound was closed leaving the distractor exposed for access. Sutures 
were removed 14 days postoperatively. At 3 postoperative weeks, the distraction was initiated four times per day with $0.25-\mathrm{mm}$ movement at each time interval. The distraction period lasted 15 days followed by a 12-week period of healing to enable consolidation of the bone in the distracted area of the mandible. After confirming good bone formation with radiographic examination, the ADO device was removed. After completing bone augmentation, implant placement could be performed in this ADO area.

\section{Implant Placement}

Approximately 4 to 5 months after bone augmentation, 100 implants (20 ZIs and 80 CIs) were placed. Before implant placement, surgical guides were completed to improve the precision of implant placement based on computerized tomographic scanning. Under general anesthesia, 20 ZIs and 40 CIs were placed in the maxilla, and $40 \mathrm{CIs}$ were placed in the mandible. Briefly, after the surgical guides were stabilized in the alveolar bone, the precise position of implant placement was demarcated with a long drill, and the soft tissue was reflected. For ZI placement, the ZI location was defined according to the surgical guide, and a channel or slot was created to define the orientation of the trajectory of the drills using a round bur. Next, the ZI osteotomies were created. At the ZI location, a 2.9-mm drill bit and a 2.9-mm twist drill were used to define the ZI position. Then, a 3.5-mm pilot drill and 3.5-mm twist drill were used to expand the osteotomy. Finally, the ZIs (Brånemark system) were installed in the alveolar bone and zygoma, and the sizes were $4.0 \mathrm{~mm}$ in diameter and 35 to $52.5 \mathrm{~mm}$ in length. For CI placement, according to the surgical guide, the CIs were placed in the area of the anterior teeth of the jaw. The CIs measured $4.1 \mathrm{~mm}$ in diameter and 10 to $12 \mathrm{~mm}$ in length (ITI SLA surfaced regular neck, Institute Straumann AG, Basel, Switzerland) or $4.3 \mathrm{~mm}$ in diameter and 10 to $13 \mathrm{~mm}$ in length for the Nobel Biocare system (Nobel Biocare, Göteborg, Sweden). GBR was used to ensure effective osseointegration and to achieve an appropriate implant ratio when insufficient bone was generated after implant placement. After implant placement, the soft tissue was approximated and primarily closed with sutures. The day after surgery, the location, direction, and safety of ZIs and CIs were evaluated with panoramic radiographs and computed tomographic scans. Sutures were removed 7 to 14 days postoperatively.

\section{Prosthesis}

From 3 to 6 months following implant placement, an acrylic transitional denture was fabricated. The definitive final prostheses were completed 6 months following fabrication of the transitional denture. As previously reported, ${ }^{21}$ Computer Aided Design/Computer Aided Manufacturing (CAD/CAM) technology was used to assist in fabricating the final prosthesis. Briefly, after completing the resin pattern framework with rubber silicone, the digital data were acquired with a Procera Forte scanner (Nobel Biocare, Benelux B.V., Houten, Utrecht, The Netherlands) following the manufacturer's instructions. The data from the approved scan were then submitted to the dental laboratory (Nobel Biocare AB Box 5190, 40226 Västra Hamngatan 1, 411 17, Göteborg, Sweden).

After approximately 3 weeks, an implant-retained trial base with light polymerizing acrylic resin and wax was obtained. Next, the verification device was evaluated intraorally, and all segments were connected with autopolymerizing acrylic resin. At this time, indices of the implant-retained trial base, including esthetics, function, and occlusal vertical dimension, were evaluated and adjusted. Additionally, patients were directly involved in the tooth selection process for the prosthesis. The implant-retained trial base with wax was returned to the dental laboratory to set the selected prosthetic teeth. After evaluating the setup intraorally for esthetics, function, and occlusion, the necessary adjustments were made, and the transitional prosthesis was fabricated and delivered. Patients were placed on a standard oral hygiene regimen using chlorhexidine rinse and regular periodontal maintenance with recall visits at 1,3 , and 6 months. After 6 months, the definitive denture was fabricated and delivered on the custom-milled CAD/CAM titanium bar. Patients were given home care instructions and directed to schedule periodic maintenance appointments.

\section{Evaluation Criteria}

Three years following delivery of the final prosthesis, follow-up visits were performed. A number of indices were recorded at this time to evaluate four general clinical parameters: (1) peri-implant soft tissue evaluation included measures of probing depth (PD), sulcular bleeding index (SBI), plaque index (PI), and gingival index (GI); (2) hard tissue evaluation included evaluation of periapical radiographs and crestal bone height 
measurements of both the mesial and distal aspects of the implant; (3) evaluation of the prostheses included prosthodontic complications and repairs, such as abutment screw loosening; fracture of implants or implant loss, marginal fit of the prosthesis; and need for denture rebasing; and (4) evaluations of patient-centered variables included patient-reported changes in psychological status, as previously described, ${ }^{9,22}$ and patient satisfaction with esthetics, prosthesis comfort, ability to enunciate, feelings of self-image, and any other psychosocial factors.

\section{Statistical Analysis}

The study data were analyzed with SPSS software version 10.0 (SPSS Inc., Chicago, IL, USA). Cohen's kappa ( $\kappa)$ test was used to measure the interexaminer reliability, and the kappa $(\kappa)$ values were greater than 0.8 . The $p$ values for multiple testing were not adjusted for the descriptive characteristics of the study.

\section{RESULTS}

All patients were treated according to the protocol, as outlined in Table 2. Ten ED patients with edentulism received 100 dental implants (20 ZIs and $80 \mathrm{CIs}$ ) between January 2007 and April 2011. ADO was used to successfully augment bone volume in the mandible for all patients. Six patients chose iliac grafts for augmentation in the maxilla, while fibula grafts were chosen by four patients. There was evidence of significant bone graft resorption in the maxilla for four patients (three autogenous ilium bone grafts and one fibula graft). Three months after the implant placements, nine CIs failed and were removed in the anterior region of the maxilla in four ED patients. Nineteen implantsupported fixed dentures and one implant-supported overdenture were placed in $10 \mathrm{ED}$ patients with edentulism (Table 1). All patients were followed for 3 years.

All ZIs were successful, and the CI success rates were $77.50 \%$ in the maxilla and $100 \%$ in the mandible (mean $88.75 \%$ ). None of the implants failed postloading during the 3 years follow-up. Periapical films demonstrated that bone resorption ranged from $1.3 \pm 0.4 \mathrm{~mm}$ to $1.8 \pm 0.6 \mathrm{~mm}$ during the follow-up period (Table 3 ). The results of the PD, SBI, PI, and GI analyses postloading are shown in Table 4. A PD measurement of 0 to $3 \mathrm{~mm}$ was observed in $43 \%$ of the patients in the first year of follow-up postloading and decreased to $29 \%$ of the patients by the third follow-up year postloading. A BI score of 0 was measured in $61 \%$ of the patients in the first year and $55 \%$ by the third year. The prevalence of a PI score of 1 increased slightly from $22 \%$ during the first year to $35 \%$ by the third year of follow-up. In addition, the proportion of patients with a GI value of 1 increased from $33 \%$ during the first year to $37 \%$ in the third year. There were four cases of peri-implant gingival hyperplasia during the follow-up period. Three of these cases were treated surgically with a gingivectomy procedure, and satisfactory clinical results were obtained after 1 year. In the third year, one case again exhibited slight gingival hyperplasia. However, no notable PD changes were observed, and bone resorption was not observed in these cases during the follow-up period (Table 3). Few prosthetic complications were recorded for the implantsupported overdenture case during the 3-year follow-up. A total of three maintenance procedures were completed for this case including two procedures in the first year and one in the third year.

Most patients were completely satisfied with the esthetics and function of their prosthesis, yet two patients were only partially satisfied (facial contours, $n=1$, and speech enunciation, $n=1$ ) (Table 5).

\begin{tabular}{lcc}
\hline TABLE 2 Patients' Treatment Protocol of the Study & \\
\hline Bone Augmentation & Implant & Prosthesis \\
\hline $\begin{array}{l}\text { Maxilla } \\
\text { Autologous bone graft (ilium or }\end{array}$ & Maxilla & Maxilla \\
fibula) + GBR & 2 ZIs and 4 CIs & Implant-supported fixed dentures \\
Mandible & Mandible & Mandible \\
ADO + GBR & 4 CIs & Implant-supported fixed dentures \\
& 4 CIs & Implant-supported overdenture
\end{tabular}

$\mathrm{ADO}=$ alveolar distraction osteogenesis; $\mathrm{CI}=$ conventional implant; GBR = guided bone regeneration; $\mathrm{ZI}=$ zygomatic implant. 


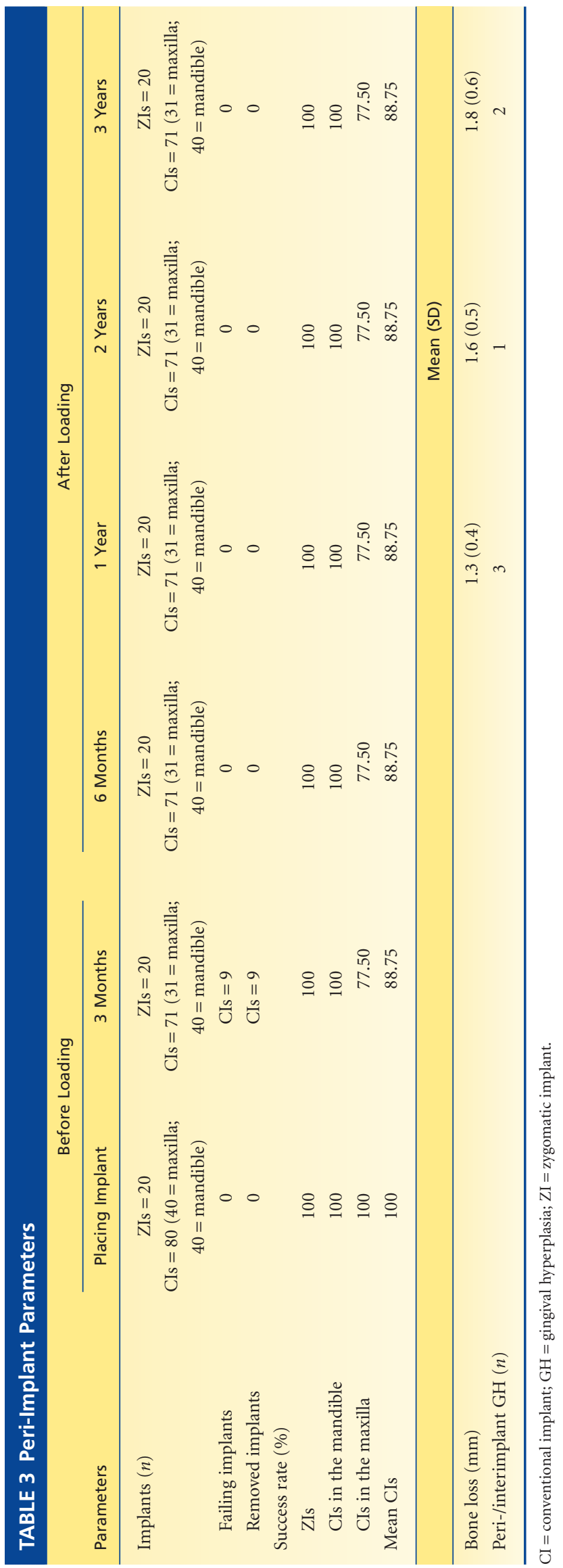

\begin{tabular}{|c|c|c|c|}
\hline Parameters & 1 year & 2 years & 3 years \\
\hline Implant $(n)$ & 91 & 91 & 91 \\
\hline \multicolumn{4}{|c|}{ Probing depths (PD) (\%) } \\
\hline Depth $=0-3 \mathrm{~mm}$ & 43 & 36 & 29 \\
\hline Depth $=3-5 \mathrm{~mm}$ & 54 & 62 & 68 \\
\hline Depth > $5 \mathrm{~mm}$ & 3 & 2 & 3 \\
\hline \multicolumn{4}{|c|}{ Modified plaque index (\%) } \\
\hline Score $=0$ & 67 & 59 & 55 \\
\hline Score $=1$ & 22 & 33 & 35 \\
\hline Score $=2$ & 11 & 8 & 10 \\
\hline Score $=3$ & 0 & 0 & 0 \\
\hline \multicolumn{4}{|c|}{ Bleeding index (BI) (\%) } \\
\hline Score $=0$ & 61 & 54 & 55 \\
\hline Score $=1$ & 36 & 45 & 42 \\
\hline Score $=2$ & 3 & 1 & 3 \\
\hline Score $=3$ & 0 & 0 & 0 \\
\hline \multicolumn{4}{|c|}{ Gingival index (GI) (\%) } \\
\hline Score $=0$ & 62 & 57 & 58 \\
\hline Score $=1$ & 33 & 42 & 37 \\
\hline Score $=2$ & 2 & 0 & 2 \\
\hline Score $=3$ & 3 & 1 & 3 \\
\hline
\end{tabular}

Following restoration of oral function with the oral reconstructions, psychological changes in all patients were recorded: (1) six patients experienced relief from depression; (2) seven patients experienced an enhancement in self-image and six patients had an increase in self-esteem; (3) social self-confidence levels of five patients were enhanced; and (4) four patients were able to obtain jobs, while six patients were able to engage in intimate interpersonal relationships following therapy (Table 5). The restorative outcome of one case is illustrated in Figure 1.

\section{DISCUSSION}

The severe lack of bone makes it challenging to restore oral function in edentulous ED adults. The clinical challenge in treating these cases was the planning and sequencing of the surgical, prosthetic, and educational steps for the predictable management of the patient's functional, esthetic, and psychological needs. ${ }^{23}$ Therefore, it is necessary to use a comprehensive and systematic protocol for psychological and oral health education, bone augmentation, placement of ZIs and CIs, and fabrication of transitional and definitive pros- 


\begin{tabular}{|c|c|c|}
\hline $\begin{array}{l}\text { Parameters } \\
\text { Patients ( } n)\end{array}$ & $\begin{array}{l}\text { Before Oral Function } \\
\text { Reconstruction } \\
10\end{array}$ & $\begin{array}{l}\text { After Oral Function } \\
\text { Reconstruction } \\
10\end{array}$ \\
\hline \multicolumn{3}{|l|}{ Patient satisfaction } \\
\hline \multirow[t]{2}{*}{ Facial contour } & 10 patients $=0$ & 9 patients $=2$ \\
\hline & & 1 patient $=1$ \\
\hline Prosthesis esthetics & & 10 patients $=2$ \\
\hline Prosthesis function & & 10 patients $=2$ \\
\hline \multirow[t]{2}{*}{ Pronunciation } & 9 patients $=0$ & 8 patients $=2$ \\
\hline & 1 patient $=1$ & 2 patients $=1$ \\
\hline \multicolumn{3}{|l|}{ Change of psychology } \\
\hline Depression & 9 patients & 3 patients \\
\hline Self-image & 10 patients $=$ Poor & 3 patients $=$ Poor \\
\hline Self-esteem & 9 patients $=$ Poor & 3 patients $=$ Poor \\
\hline Social self-confidence & 9 patients $=$ Poor & 4 patients $=$ Poor \\
\hline Partner & 1 patient & 7 patients \\
\hline Vocational factors & 0 patient & 4 patients \\
\hline
\end{tabular}

$0=$ unsatisfied; $1=$ partially satisfied; $2=$ fully satisfied .

theses for these patients. This prospective study demonstrates the effectiveness of restoring oral function in edentulous ED patients with severely insufficient bone volume using ZI and CI therapy combined with bone augmentation.

Since Brånemark's osseointegration theory was presented in 1982, CIs have been successfully used to restore oral function to patients with tooth loss. In 1998, the use of ZIs (fixed in the lateral orbital rim and zygomatic arch) was first reported. ${ }^{8}$ ZIs provide a good alternative to rehabilitate patients with a severely atrophic maxilla, without the need for performing bone augmentation procedures. In adult edentulous ED patients, severe atrophy of the alveolar ridge is the biggest challenge in
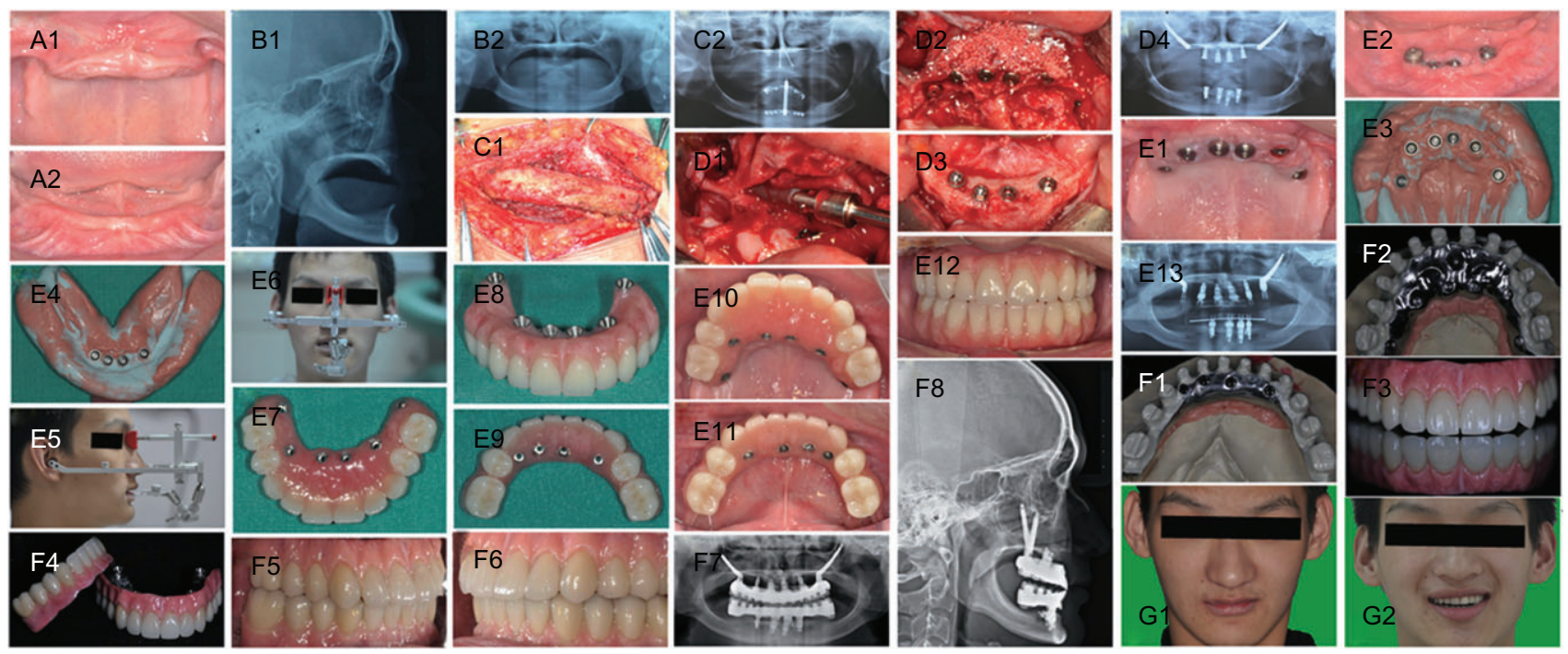

Figure 1 Clinical procedure using ZI- and CI-supported fixed prosthetic replacements to restore oral function in adult ED patients with anodontia. $A 1-B 2$, An evaluation of the patient's characteristics using intraoral and panoramic X-rays. C1-C2, Bone augmentation using autogenous bone grafting from the ilium and DO. D1-D4, Implant placement. E1-E11, Implant-supported fixed transitional prosthetic rehabilitation. F1-F8, Implant-supported fixed formal prosthetic rehabilitation with CAD/CAM technology. G1-G2, Frontal pictures before and after the reconstruction. 
restoring oral function. In this study, we chose several techniques to augment the bone volume including $\mathrm{ADO}$, autogenous bone grafts, and artificial bone grafts. $\mathrm{ADO}$ was used to address bone height deficiencies, while autogenous and allogeneic grafting was used to address deficiencies in bone width. Bone augmentation was successful in the mandible for all patients; however, four patients experienced notable bone resorption in the maxilla. In 2002, Guckes showed implant success rates in adult ED patients of $95 \%$ for the maxilla and $97 \%$ for the mandible. ${ }^{24}$ These data provide good evidence for the restoration of oral function in ED patients using dental implants. A more recent study evaluated the bone microarchitecture at oral implant sites in ED patients, and the results showed that the bone of females with ED was more compact and had greater trabecular connectedness than the bone from males with $\mathrm{ED} .^{25}$ These findings associated with the gender differences in bone of ED patients may influence treatment options when considering bone augmentation and implant therapy in ED patients. In our study, implant success rates for ED patients were the same as that seen in systemically healthy patients, and there were no differences in success rates associated with gender.

In our study, nine CIs failed and were removed 3 months after implant placement in four patients with iliac or fibular grafts. The reasons for implant failure are most likely related to bone graft resorption in the anterior region of the maxilla. Several factors may play a role in this result: (1) greater bone resorption of iliac grafts compared with other autogenous bone graft donor sites $^{26}$; (2) insufficient vascularization to support maintenance of the graft due to the severe atrophy of the grafted area; (3) compared with normal patients, the alveolar bone in the anterior region of the maxilla of adult edentulous ED patients has more "fibrous bone," and this condition counteracts bone grafting and the osseointegration of implants.

All ZIs were successful, and no complications associated with peri-implantitis, infections, maxillary sinusitis, and the orbital rim were identified. Four patients presented with hematoma around the eyes 24 to 48 hours after their ZI placement surgeries, but their eyesight remained normal. After 1 week, the hematoma disappeared. After loading, during the 3-year follow up period, the three main clinical problems identified were (1) cheek biting of the buccal mucosa, (2) calculus accumulation, and (3) gingival hyperplasia. Prior to therapy,
10 ED patients in this study had never had a dentition, making it very difficult for them to adapt to a fully restored dentition. With oral health education and adjustments to the definitive prostheses, cheek biting was not frequently noted beyond 6 months postloading of the definitive prosthesis. Oral hygiene education was also important in these patients, and though calculus accumulation was still noted throughout the course of the 3-year follow-up, its prevalence decreased following the 1 year follow-ups with consistent patient education resulting in more diligent patient home care. Four cases of gingival hyperplasia occurred (one case in the maxilla, three cases in mandible) over 3 years, and in one case, the gingival hyperplasia disappeared 7 to 10 days after the calculus was removed. Three cases (one case in the maxilla, two cases in mandible) were treated with a gingivectomy, and good results were obtained. However, one patient experienced postsurgical recurrence (in the mandible). The causative factors that could contribute to gingival hyperplasia include hormonal changes, etiology, chronic inflammation, and certain diseases (leukemia, especially monocytic), as well as the use of certain drugs (phenytoin, cyclosporine, nifedipine, and other calcium channel blockers). ${ }^{27}$ In this study, gingival hyperplasia may be caused by chronic inflammation and minimal attached and keratinized gingiva around the implants. Chronic inflammation was associated with local factors, such as plaque, calculus, bacteria, or other unknown factors. ${ }^{28}$ Previous reports showed that skin grafts or free gingival grafts from the palate around can inhibit or reduce inflammation and prevent gingival hyperplasia. ${ }^{29}$

Based on the above data, oral hygiene played a critical role in the prevention of gingival hyperplasia and even peri-implantitis in the successful restoration of the oral function of the patients in the present study. Hygiene measures, as previously described, ${ }^{9}$ include the following: the correct use of a toothbrush, dental floss, and a "WaterPik"; compliance with follow-up; adherence to recommended periodontal maintenance schedules; and supervision or assistance from family members in maintaining the patient's oral hygiene.

All patients were completely satisfied with the esthetics and function of the prosthesis. The unique facial features of ED patients exacerbate the social challenge of maintaining self-confidence and having healthy social interactions. ${ }^{30}$ Almost all ED patients in this study reported initial low self-esteem, speech defi- 
ciencies, decreased academic performance, and social isolation; these factors can contribute to inappropriate social behaviors and impaired social interactions. ${ }^{30}$ Therefore, it is important to help patients enhance their self-esteem and social recognition. In this study, psychological education was evaluated, and positive results were obtained. Over $50 \%$ of the patients reported psychological and social improvements associated with depression, self-image, social self-confidence, and the ability to obtain jobs and intimate relational partners.

Previous studies have described protocols for restoring oral function to ED patients. Yet these studies are primarily case reports. ${ }^{31-39}$ Few reports have demonstrated the effective treatment of adult edentulous ED patients with severe bone atrophy in the jaw using both ZIs and CIs. Additionally, no reports have described the use of a comprehensive and systematic treatment protocol to restore oral function for these patients while addressing the psychological and educational barriers and challenges associated with their treatment. Though this was a prospective evaluation of this treatment protocol, given the limited number of cases and time of follow-up, longer-term evaluations of implant success rates and complications are needed.

\section{CONCLUSIONS}

The main objective of this study was to establish a comprehensive, systematic, and effective measure for restoring oral function in adult ED patients with a severe lack of bone. This study determined that bone augmentation using $\mathrm{ADO}$ and autogenous bone grafting is a promising method to provide sufficient bone volume for implant placement. However, bone augmentation of the maxilla has limitations when using autogenous bone derived from extraoral donor sites due to resorption, and it is necessary to find an effective method to resolve this challenge. Additionally, considering the limited study time and number of cases, it is necessary to further validate these mentioned conclusions in future studies that include a greater number of clinical cases and a longer follow-up period.

\section{ACKNOWLEDGEMENTS}

This study was funded by the Combined Engineering and Medical Project of Shanghai Jiao Tong University (YG2012MS32), the Science and Technology Commission of Shanghai Municipality (13ZR1424000), the National Natural Science Foundation of China
(31370983, 81371190), the Key Project of the Chinese Ministry of Education (212080), Grants for Scientific Research of BSKY(XJ201109), and the Young Top-Notch Talent Support Scheme from Anhui Medical University.

\section{REFERENCES}

1. Pigno MA, Blackman RB, Cronin RJ Jr, et al. Prosthodontic management of ectodermal dysplasia: a review of the literature. J Prosthet Dent 1996; 76:541-545.

2. Siponen M, Neville BW, Damm DD, et al. Multifocal lateral periodontal cysts: a report of 4 cases and review of the literature. Oral Surg Oral Med Oral Pathol Oral Radiol Endod 2011; 111:225-233.

3. Bergendal B. Oligodontia ectodermal dysplasia-on signs, symptoms, genetics, and outcomes of dental treatment. Swed Dent J Suppl 2010; 13-78:7-8.

4. Van Sickels JE, Raybould TP, Hicks EP. Interdisciplinary management of patients with ectodermal dysplasia. J Oral Implantol 2010; 36:239-245.

5. NaBadalung DP. Prosthodontic rehabilitation of an anhidrotic ectodermal dysplasia patient: a clinical report. J Prosthet Dent 1999; 81:499-502.

6. Liu Y, Yu X, Wang L, et al. Mutation p.Leu354Pro in EDA causes severe hypohidrotic ectodermal dysplasia in a Chinese family. Gene 2012; 491:246-250.

7. Yenisey M, Guler A, Unal U. Orthodontic and prosthodontic treatment of ectodermal dysplasia-a case report. Br Dent J 2004; 196:677-679.

8. Penarrocha-Diago M, Uribe-Origone R, Rambla-Ferrer J, et al. Fixed rehabilitation of a patient with hypohidrotic ectodermal dysplasia using zygomatic implants. Oral Surg Oral Med Oral Pathol Oral Radiol Endod 2004; 98:161-165.

9. Zou D, Wu Y, Wang XD, et al. A retrospective 3-to-5 year study of the reconstruction of oral function using implantsupported prostheses in patients with hypohidrotic ectodermal dysplasia. J Oral Implantol 2014; 40:571-580.

10. Cheung LK, Hariri F, Chua HD. Alveolar distraction osteogenesis for oral rehabilitation in reconstructed jaws. Curr Opin Otolaryngol Head Neck Surg 2011; 19:312-316.

11. Chin M, Toth BA. Distraction osteogenesis in maxillofacial surgery using internal devices: review of five cases. J Oral Maxillofac Surg 1996; 54:45-53.

12. Cano J, Campo J, Moreno LA, et al. Osteogenic alveolar distraction: a review of the literature. Oral Surg Oral Med Oral Pathol Oral Radiol Endod 2006; 101:11-28.

13. Huang W, Wu Y, Zou D, et al. Long-term results for maxillary rehabilitation with dental implants after tumor resection. LID. Clin Implant Dent Relat Res 2014; 16:282-291.

14. Mitchell DA, Macleod SP. Strategies for avoiding complications with vascularized bone flaps in head and neck microvascular reconstruction. Semin Plast Surg 2008; 22:175-185.

15. Bayat M, Khobyari MM, Dalband M, et al. Full mouth implant rehabilitation of a patient with ectodermal dysplasia 
after orthognathic surgery, sinus and ridge augmentation: a clinical report. J Adv Prosthodont 2011; 3:96-100.

16. Rad AS, Siadat H, Monzavi A, et al. Full mouth rehabilitation of a hypohidrotic ectodermal dysplasia patient with dental implants: a clinical report. J Prosthodont 2007; 16:209-213.

17. Kirmeier R, Gluhak C, Marada P, et al. Oral rehabilitation of adult twins with severe lack of bone due to hypohidrotic ectodermal dysplasia-a 12-month follow-up. J Oral Maxillofac Surg 2009; 67:189-194.

18. Grecchi F, Pagliani L, Mancini GE, et al. Implant treatment in grafted and native bone in patients affected by ectodermal dysplasia. J Craniofac Surg 2010; 21:1776-1780.

19. Malo P, de Araujo Nobre M, Lopes A, et al. Extramaxillary surgical technique: clinical outcome of 352 patients rehabilitated with 747 zygomatic implants with a follow-up between 6 months and 7 years. LID. Clin Implant Dent Relat Res 2013. 10.1111/cid.12147 [doi].

20. Li D, Liu Y, Ma W, et al. Review of ectodermal dysplasia: case report on treatment planning and surgical management of oligodontia with implant restorations. Implant Dent 2011; 20:328-330.

21. Lin WS, Harris BT, Zandinejad A, et al. Use of digital data acquisition and CAD/CAM technology for the fabrication of a fixed complete dental prosthesis on dental implants. J Prosthet Dent 2014; 111:1-5.

22. Hickey AJ, Salter M. Prosthodontic and psychological factors in treating patients with congenital and craniofacial defects. J Prosthet Dent 2006; 95:392-396.

23. Dhima M, Salinas TJ, Cofer SA, et al. Rehabilitation of medically complex ectodermal dysplasia with novel surgical and prosthodontic protocols.LID. Int J Oral Maxillofac Surg 2013. 10.1016/j.ijom.2013.08.004 [doi]; S0901-5027(13) 01071-0 [pii]LID.

24. Guckes AD, Scurria MS, King TS, et al. Prospective clinical trial of dental implants in persons with ectodermal dysplasia. J Prosthet Dent 2002; 88:21-25.

25. Silthampitag $\mathrm{P}$, Klineberg I, Austin B, et al. Bone microarchitecture at oral implant sites in ectodermal dysplasia (ED): a comparison between males and females. Clin Oral Implants Res 2012; 23:1275-1282.

26. Tiwana PS, De Kok IJ, Stoker DS, et al. Facial distortion secondary to idiopathic gingival hyperplasia: surgical management and oral reconstruction with endosseous implants. Oral Surg Oral Med Oral Pathol Oral Radiol Endod 2005; 100:153-157.

27. Santamaria MP, Casati MZ, Nociti FH Jr, et al. Connective tissue graft plus resin-modified glass ionomer restoration for the treatment of gingival recession associated with non- carious cervical lesions: microbiological and immunological results. Clin Oral Investig 2013; 17:67-77.

28. Ciocca L, Corinaldesi G, Marchetti C, et al. Gingival hyperplasia around implants in the maxilla and jaw reconstructed by fibula free flap. Int J Oral Maxillofac Surg 2008; 37:478480.

29. Macgregor FC. Facial disfigurement: problems and management of social interaction and implications for mental health. Aesthetic Plast Surg 1990; 14:249-257.

30. Pope AW, Ward J. Factors associated with peer social competence in preadolescents with craniofacial anomalies. J Pediatr Psychol 1997; 22:455-469.

31. Pombo CM, Luaces RR, Arenaz BJ, et al. Prosthodontic rehabilitation in patient with ectodermal dysplasia combining preprosthetic techniques: a case report. Implant Dent 2013; 22:460-464.

32. Singer SL, Henry PJ, Liddelow G, et al. Long-term follow-up of implant treatment for oligodontia in an actively growing individual: a clinical report. J Prosthet Dent 2012; 108:279285.

33. Al-Ibrahim HA, Al-Hadlaq SM, Abduljabbar TS, et al. Surgical and implant-supported fixed prosthetic treatment of a patient with ectodermal dysplasia: a case report. Spec Care Dentist 2012; 32:1-5.

34. Penarrocha M, Garcia B, Marti E, et al. Rehabilitation of severely atrophic maxillae with fixed implant-supported prostheses using zygomatic implants placed using the sinus slot technique: clinical report on a series of 21 patients. Int J Oral Maxillofac Implants 2007; 22:645-650.

35. Alcan T, Basa S, Kargul B. Growth analysis of a patient with ectodermal dysplasia treated with endosseous implants: 6-year follow-up. J Oral Rehabil 2006; 33:175-182.

36. Suri S, Carmichael RP, Tompson BD. Simultaneous functional and fixed appliance therapy for growth modification and dental alignment prior to prosthetic habilitation in hypohidrotic ectodermal dysplasia: a clinical report. J Prosthet Dent 2004; 92:428-433.

37. Rashedi B. Prosthodontic treatment with implant fixed prosthesis for a patient with ectodermal dysplasia: a clinical report. J Prosthodont 2003; 12:198-201.

38. Bergendal B. Prosthetic habilitation of a young patient with hypohidrotic ectodermal dysplasia and oligodontia: a case report of 20 years of treatment. Int J Prosthodont 2001; 14:471-479.

39. Pavarina AC, Machado AL, Vergani CE, et al. Overlay removable partial dentures for a patient with ectodermal dysplasia: a clinical report. J Prosthet Dent 2001; 86:574-577. 